\title{
Chemically Polished Aluminum Alloys with Low Outgassing Rate
}

\author{
Sakae INAYOSHI*, Kazuya SAITO*, Sonoko TSUKAHARA*, \\ Katsunobu ISHIZAWA**, Takeshi NOMURA** and Minoru KANAZAWA**** \\ ${ }^{*}$ Tsukuba Institute for Super Materials ULVAC JAPAN, Ltd., 5-9-7, Tokodai, Tsukuba, Ibaraki \\ ${ }^{* *}$ San-ai OIL, Ltd., and ${ }^{* * *}$ San-ai Plant Construction, Ltd., \\ 3-27-1, Tonomachi, Kawasaki, Kawasaki, Kanagawa
}

(Received January 7, 1998, Accepted May 2, 1998)

\begin{abstract}
A technique of chemical polishing and subsequent cleaning was developed as the final surface treatment of most of aluminum alloys those are used as component materials in ultrahigh and extremely high vacuum equipments. This paper focuses to most recurrently used alloys, A5052 and A6061. Surfaces treated by the new technique are smooth enough to give a clear reflection. The outgassing properties by thermal desorption spectroscopy, pump-down characteristics, and surface analyses by Auger electron spectroscopy, atomic force microscopy, Brunauer-Emmett-Teller method, and humidity cabinet test were examined on the chemically polished samples. The outgassing rate of the chemically polished samples is in the lowest range of value to those reported for mechanically machined aluminum alloys. The atmospheric corrosion resistance of chemically polished samples is better than that of any mechanically machined samples, and the specially treated chemically polished samples has higher corrosion resistance than the electrolytically polished samples.
\end{abstract}

\section{Introduction}

Aluminum alloys are commonly used materials for chambers and components of ultrahigh vacuum systems. Several surface treatment techniques of aluminum alloys such as special extrusion $(E X)^{1)}$, ethanol lathing $(E L)^{2}$, and chemical cleaning 3 ,4) have been already developed to lower outgassing rate.

As a new useful treatment of the aluminum alloys for a vacuum material application purpose here we propose another technique, chemical polishing, which had been used as a conventional surface treatment of metals including aluminum alloys for many purposes, but not for vacuum material use. The technique consists of chemical polishing and subsequent cleaning and is applicable to nearly all the aluminum alloys used in vacuum system. A wet process has an advantage to be able to treat materials wholly in any shape at the final step of parts fabrication process and that is an important point in practical applications.

In the first step of chemical polishing experiment of aluminum alloys all the alloys used in vacuum equipments were examined and nearly the same results were obtained as reported previously5). As the next step this paper focuses to the most recurrently used alloys, A5052 and A6061. Outgassing rates of the resultant specimen were examined and compared with those of the samples treated by conventional and previously developed techniques. The low outgassing rate of chemically polished samples was consistent with the results of surface analysis such as atomic force microscopy (AFM), Auger electron spectroscopy (AES), and Brunauer-Emmett-Teller (BET) method').

\section{Experimental}

\subsection{Samples}

Aluminum alloys of A5052 and A6061 were examined. Samples size was changed depending on measurement technique as noted below.

A5052 samples of $\phi 45 \mathrm{~mm} \times 3 \mathrm{~mm}$ (surface area $S=3.6 \times 10^{-3} \mathrm{~m}^{2}$ ) were used for thermal desorption spectroscopy (TDS) and surface analysis purpose. Five differently treated samples were prepared through three steps of treatments. At the first step a rod was shaped out from block material and was sliced to plates by conventional machining, which process we call the first mechanical 


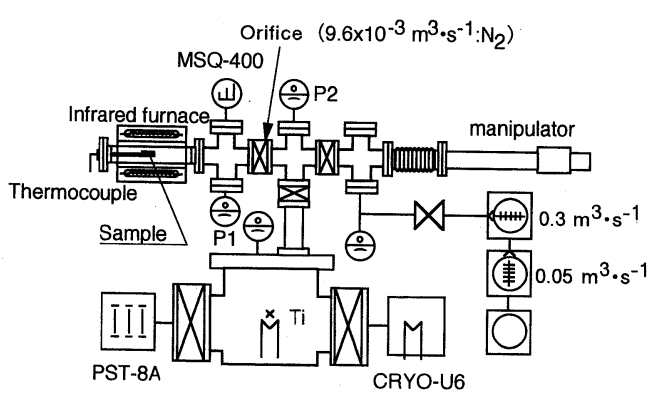

Fig. 1 Configuration of the thermal desorption spectroscopy system.

machining (MM1). Then in the second step all the surfaces of each MM1 sample were carefully lathed to have almost the same roughness, which we call the second mechanical machining (MM2). In the third step the MM2 sample was treated by an additional treatment such as chemical polishing $(\mathrm{CP})$, electrolytic polishing (EP), or ethanol lathing $(E L)^{2}$. For some cases samples were EX treated ${ }^{7}$; machined with blowing $\mathrm{O}_{2}$ and $\mathrm{Ar}$ on works, in place to EL.

Each five pieces of $\mathrm{CP}$ and EP finished plates of $200 \mathrm{~mm} \times 250 \mathrm{~mm} \times 2 \mathrm{~mm}, S=0.5 \mathrm{~m}^{2}$ in total, were used as the large area samples of A5052 and A6061 alloys for pump-down characteristic measurement.

Two chamber samples of $\phi 254 \mathrm{~mm} \times 380 \mathrm{~mm}$ long $\left(S=0.4 \mathrm{~m}^{2}\right)$ with $\phi 306 \mathrm{~mm}$ metal seal flanges of A6061 alloy were prepared and each one was finished CP or EP for the measurement at the ultimate pressure.

\subsection{Final treatment}

Two kinds of chemical polishing solutions with phosphoric acid and nitric acid as the main ingredient, San-ai CP1000 and San-ai CP2000X, were developed ${ }^{5}$. The former or the latter was used for polishing aluminum alloys of A1000, A5000, and A6000 series or A2000 series, respectively.

All the aluminum samples from small plates to large chamber were chemically polished by directly dipping into the polishing solution.

Various parameters of the chemical polishing and cleaning with hot and cold pure water immediately after the chemical polishing were examined to obtain the optimum condition for each series of alloys $^{5)}$. About $4 \mu \mathrm{m}$ of the specimen surface was polished at the standard treatment condition described in this paper. When it is necessary for special purpose, thickness of the polished layer can be increased up to $40 \mu \mathrm{m}$ keeping the vacuum proper-

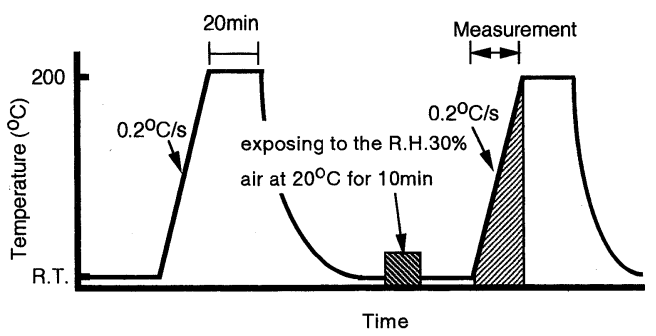

Fig. 2 Temperature-Time table of the TDS experiment.

ty of the treated samples the same.

To obtain typical EP, EL, and EX samples we ordered the similarly prepared MM2 state samples to be treated by commercially available techniques. The EP samples were polished by phosphoric acid solution and cleaned with city water. The EL and EX samples were machined with diamond bites.

\subsection{Outgassing rate}

Outgassing rate $q$ of small samples was measured by the thermal desorption spectroscopy (TDS) system schematically shown in Fig. 1. The sample was heated up to $200^{\circ} \mathrm{C}$ at the rate of $0.2^{\circ} \mathrm{C} / \mathrm{s}$ through a quartz glass tube with an infrared furnace that was outside the vacuum system. The pressures of both sides of an orifice were measured by two B-A gauges to give outgassing rate, where the conductance of the orifice was $9.6 \times 10^{-3} \mathrm{~m}^{3} / \mathrm{s}$. The outgassing species was detected by a quadrupole mass spectrometer in the up stream side.

All the samples were measured twice depending on the temperature-time table in Fig. 2. The first heating step for $20 \mathrm{~min}$ was necessary to initialize samples that had experienced different conditions and the second heating step after exposure of the sample to the $30 \%$ relative humidity (R.H.) air for 10 min was used for comparison of many samples in this paper.

As a practical pump-down experiment, time depending outgassing rate of large area samples were measured by a conventional throughput method, where the conductance of the orifice was $6.1 \times 10^{-3}$ $\mathrm{m}^{3} / \mathrm{s}$ after exposure of the sample to the air of $50 \%$ R.H. for $1 \mathrm{~h}$ at room temperature following to the $150^{\circ} \mathrm{C} \times 20 \mathrm{~h}$ baking in vacuum.

The static outgassing rate of the chamber at the ultimate pressure was also measured just after a $150^{\circ} \mathrm{C} \times 20 \mathrm{~h}$ baking by a modified throughput method, switching between two pumping paths (SPP), excluding the outgassing rate of gauges and walls of measuring apparatus ${ }^{8)}$ while the tempera- 


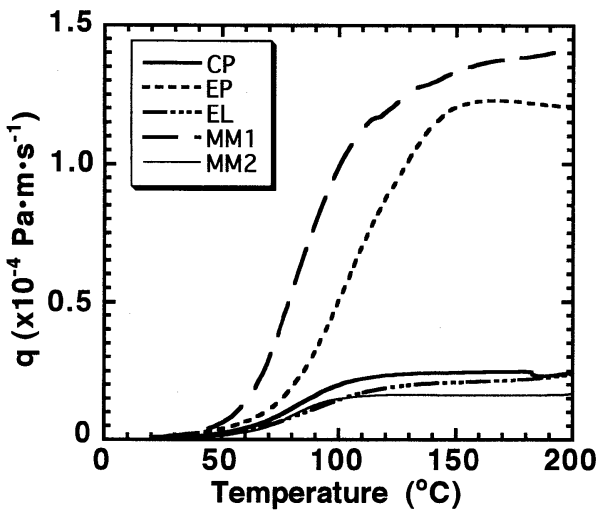

Fig. 3 Thermal desorption spectra of various surface treatments samples.

ture of the chamber was varied from room temperature to $100^{\circ} \mathrm{C}$ at every $10^{\circ} \mathrm{C}$ step.

\subsection{Surface analysis}

The microscopic and nanoscopic surface topography was observed by SEM and AFM, respectively. To detect the species and the thickness of the surface oxide layer the depth profile of samples were analyzed by AES etching with Ar from the surface at a rate of $1.5 \sim 1.7 \mathrm{~nm} / \mathrm{min}$ for aluminum layer equivalent.

Although the geometrical surfaces of all the examined samples were kept the same, the real surface area depends on the surface treatments. The real surface area of the $\mathrm{CP}$ and EP samples were measured by BET method with a self-made Watanabe-type ${ }^{9)}$ apparatus using the isotherm of $\mathrm{Xe}$ at $77 \mathrm{~K}$ after $150^{\circ} \mathrm{C} \times 16 \mathrm{~h}$ baking of the apparatus with the sample.

In BET method we compared the surface area using the mean value of effective cross-sectional area $\sigma(\mathrm{Xe})=25 \AA^{2}$ on Ni powder and glass wool ${ }^{10)}$, because the effective cross-sectional area of Xe molecule on the aluminum surface in this condition is unclear.

\subsection{Humidity cabinet test}

The humidity cabinet test of JIS2246 method was applied to all the series of alloys. Each sample differently treated put in and kept in a test chamber at $49 \pm 1{ }^{\circ} \mathrm{C}$ with more than R.H. $95 \%$ and the color-change-time that is the time until sample color is changed by corrosion was measured.

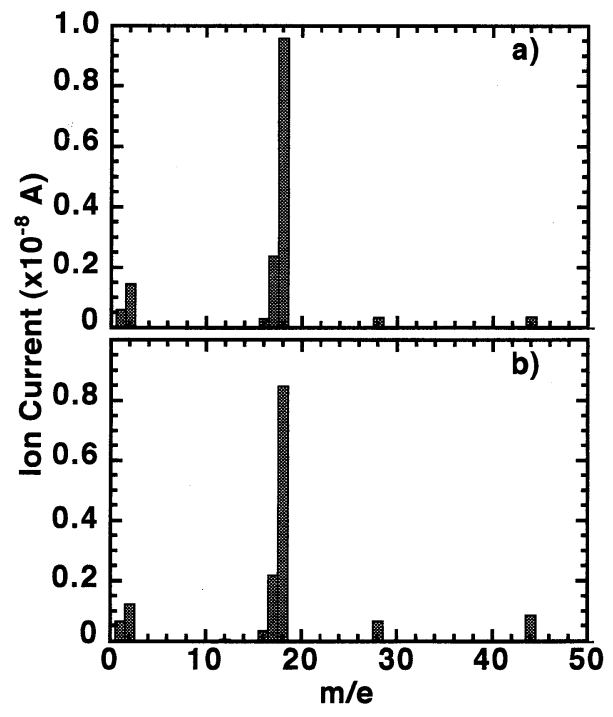

Fig. 4 Mass spectra at $200^{\circ} \mathrm{C}$ with TDS. a) CP and b) EL.

\section{Results}

\subsection{Outgassing rate}

Thermal desorption spectra of A5052 alloy samples after various surface treatments are compared in Fig. 3. The resultant spectra are separated into two groups. EP and MM1 are one group with higher $q$ up to higher temperature, whereas $\mathrm{CP}$, MM2 and EL are the other group with lower $q$. Employing the area $Q$ under each thermal desorption spectrum from room temperature to $200^{\circ} \mathrm{C}$ as a measure of outgassing amount they are compared as follows;

$$
\begin{aligned}
& Q(\mathrm{CP}): Q(\mathrm{MM} 2): Q(\mathrm{EL}): Q(\mathrm{EP}): Q(\mathrm{MM} 1) \\
& \quad=1: 0.7: 0.9: 4.1: 5.5 .
\end{aligned}
$$

It was confirmed that the outgassing rate of various aluminum alloys samples after chemical polishing were nearly the same as that of A5052 alloy.

In every case the main outgassing species that contribute the $q$ value were $\mathrm{H}_{2} \mathrm{O}(\mathrm{m} / \mathrm{e}=18), \mathrm{H}_{2}$ $(\mathrm{m} / \mathrm{e}=2), \mathrm{CO}(\mathrm{m} / \mathrm{e}=28)$, and $\mathrm{CO}_{2}(\mathrm{~m} / \mathrm{e}=44)$. The mass spectra of $\mathrm{CP}$ and EL samples in the lower $q$ group at the highest temperature of this experiment, $200^{\circ} \mathrm{C}$, are compared in Fig. 4. The ion currents of $\mathrm{H}_{2}$ and $\mathrm{H}_{2} \mathrm{O}$ of $\mathrm{CP}$ were a little more than those of the EL samples, while the ion currents of $\mathrm{CO}$ and $\mathrm{CO}_{2}$ of $\mathrm{CP}$ were a little less than those of the EL sample.

Figure 5 shows the time dependence of outgassing rate of the large area CP and EP A5052 samples during pump-down from the atmosphere at 


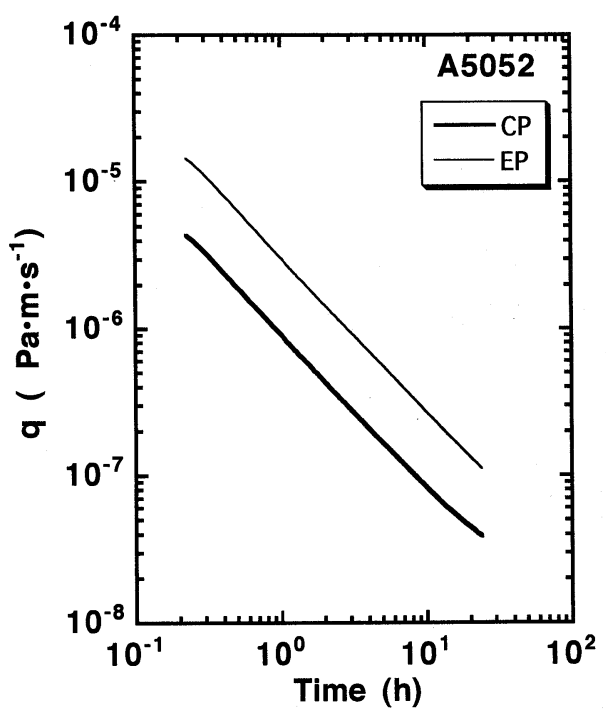

Fig. 5 Pump-down curves of large area A5052 samples at $28^{\circ} \mathrm{C}$ after exposure to the R.H. $50 \%$ air for $1 \mathrm{~h}$.

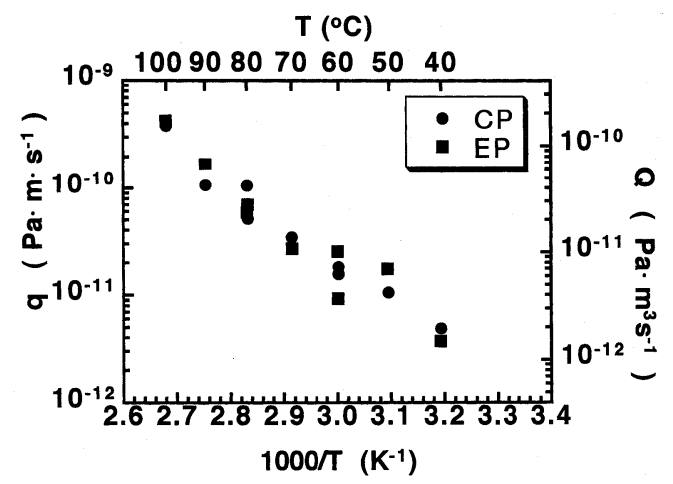

Fig. 6 Temperature dependence of the outgassing rate of $\mathrm{A} 6061$ sample chamber after $150^{\circ} \mathrm{C} \times 20 \mathrm{~h}$ baking.

room temperature. The outgassing rate of $\mathrm{CP}$ was $1 / 3$ to that of EP sample every time, where the largest ion current among above mentioned species is $\mathrm{H}_{2} \mathrm{O}$. The A6061 samples showed the same result.

Figure 6 shows temperature dependence of outgassing rates of $\mathrm{CP}$ and $\mathrm{EP}$ chamber after $150^{\circ} \mathrm{C}$ $\times 20 \mathrm{~h}$ baking measured by SPP method. The outgassing rate $q$ of $\mathrm{CP}$ chamber is $4 \sim 8 \times 10^{-12}$ $\mathrm{Pa} \cdot \mathrm{m} \cdot \mathrm{s}^{-1}$, and main residual species is $\mathrm{H}_{2}$ at room temperature. This $q$ value is low enough to satisfy the extremely high vacuum (XHV) application needs. The CP treatment is applicable to the materi-

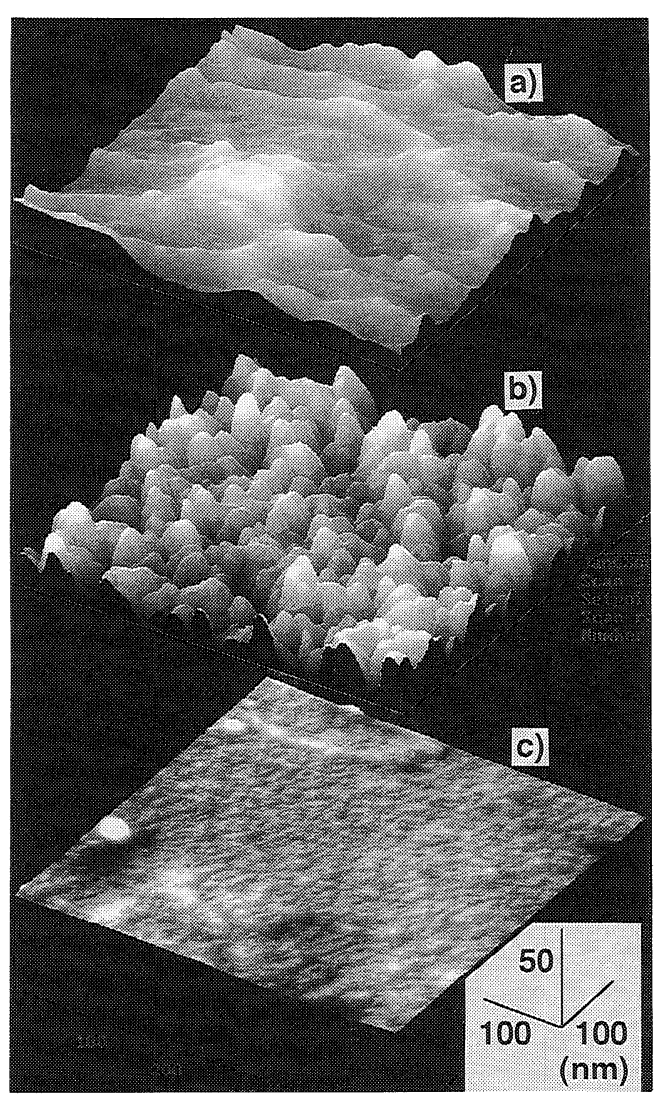

Fig. 7 Atomic force micrographs of samples. a) $\mathrm{CP}, \mathrm{b}) \mathrm{EP}$, and c) EL.

als for XHV system as well as EP.

\subsection{Surface analysis}

The CP sample showed shiny outside with clear reflection due to the decrease in surface roughness. There observed no morphological difference among the samples by SEM. However, as typical examples of atomic force micrographs of $\mathrm{CP}, \mathrm{EP}$, and EL samples of A5052 are shown in Fig. 7, the surface of EL sample are smoother than that of CP and EP had the roughest surface among them in nanoscopic scale.

The AES depth profiles of CP, EP, and EL treated A5052 samples are shown in Fig. 8. The surface oxide layer of the CP and the EL samples are a few $\mathrm{nm}$ thick and nearly the same, whereas the thickness of the oxide layer of the EP sample is about $60 \mathrm{~nm}$.

It is measured by the BET method that the number of molecules when the monolayer is completed on the CP and the EP samples were deduced as 


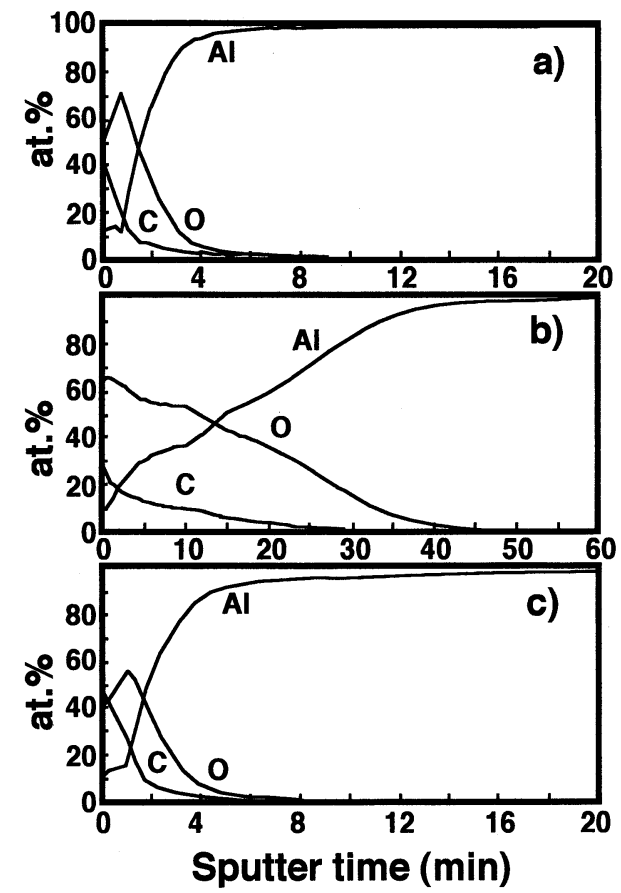

Fig. 8 Depth profiles by AES. a) CP, b) EP, and c) EL.

$8.8 \times 10^{15} \mathrm{~m}^{-2}$ and $5.0 \times 10^{16} \mathrm{~m}^{-2}$, respectively. Then the real area of the CP and the EP samples were $2.2 \times 10^{-3} \mathrm{~m}^{2}$ and $1.25 \times 10^{-2} \mathrm{~m}^{2}$, respectively.

\subsection{Humidity cabinet test}

The color of all the machined samples, MM1, MM2, and EL, changed after 1 2 h. The colorchange-time of standard CP and EP samples were $3-5 \mathrm{~h}$ and $48 \mathrm{~h}$, respectively, while it was $70 \mathrm{~h}$ after polishing the surface layer of $25 \mu \mathrm{m}$ as a special CP.

\section{Discussion}

\subsection{Outgassing rate}

The outgassing rate of $\mathrm{CP}$ samples $q_{\mathrm{CP}}$ are lower than that of EP samples $q_{\mathrm{EP}}$ both in Fig. 3 and Fig. 5 , whereas $q_{\mathrm{CP}}$ is almost equal to $q_{\mathrm{EP}}$ in Fig. 6 . The difference between those results can be explained as follows. Although in both measurements shown in Fig. 3 and Fig. 5 the main outgassing species is $\mathrm{H}_{2} \mathrm{O}$ that comes from desorption of adsorbed gas on the surface, in Fig. 6, the main residual gas species is $\mathrm{H}_{2}$ because $\mathrm{H}_{2} \mathrm{O}$ had been desorbed during $150^{\circ} \mathrm{C} \times 20 \mathrm{~h}$ baking.

To evaluate the uniformity of the $\mathrm{CP}$ and EP sur-

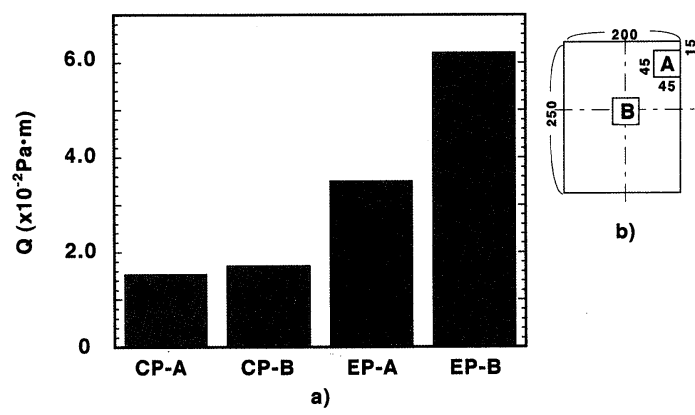

Fig. 9 a) Outgassing quantity from room temperature to $200^{\circ} \mathrm{C}$ of small samples A and B. Small samples A and B were cut off from different parts of each large area as shown in b).

face, each two small pieces of samples A and B, 45 $\mathrm{mm} \times 45 \mathrm{~mm}$ in size, were cut out from the large pump-down samples, $200 \mathrm{~mm} \times 250 \mathrm{~mm}$ in size, as shown in Fig. 9. Their outgassing $Q$, that is the area measured under a thermal desorption spectrum, such as $Q(\mathrm{CP}-\mathrm{A}), Q(\mathrm{CP}-\mathrm{B}), Q(\mathrm{EP}-\mathrm{A})$, and $Q(\mathrm{EP}-\mathrm{B})$ were compared in Fig. 9. $Q(\mathrm{CP}-\mathrm{A})$ and $Q(\mathrm{CP}-\mathrm{B})$ were almost the same, but the difference between $Q(\mathrm{EP}-\mathrm{A})$ and $Q(\mathrm{EP}-\mathrm{B})$ was more than experimental error. We extend this result to explain the above mentioned difference in the $q_{\mathrm{EP}} / q_{\mathrm{CP}}$ ratios by samples size dependence. The chemically polished surface is homogeneous because many necessary conditions for $\mathrm{CP}$ had been controlled depending on intentionally developed technique, whereas electrolytic polishing resulted nonuniform surface that might be caused by electrode arrangement.

The real surface area of CP deduced with BET method was $2.2 \times 10^{-3} \mathrm{~m}^{2}$ which is smaller than the geometrical area $3.6 \times 10^{-3} \mathrm{~m}^{2}$ because the effective cross-sectional area $\sigma(\mathrm{Xe})=25 \AA^{2}$ of $\mathrm{Ni}$ powder and glass wool might not be correct on the aluminum surface. For the numerical confirmation we must try further experimental cross-sectional area of Xe on Aluminum.

Here we would like to focus our attention to the consistency of the ratio of the real areas of EP and CP samples $1.25 \times 10^{-2} / 2.2 \times 10^{-3}=5.7$ to the ratio of outgassing of both samples 4.1 from TDS measurement in Fig. 3. This is one experimental result that the outgassing ratio cloud be explained by contribution of the surface area effect.

In AES, all the surface consist of almost the same elements as $\mathrm{Al}, \mathrm{O}$, and $\mathrm{C}$ as sown in Fig. 8, but it is well known that aluminum makes various 


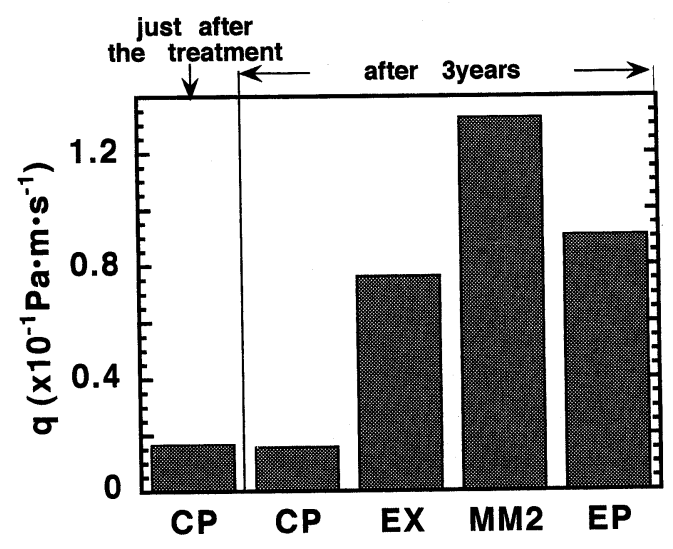

Fig. 10 Outgassing from room tenmperatuer to $200^{\circ} \mathrm{C}$ of after three years samples.

hydroxides. It must be necessary to analyze on the state of oxygen and hydrogen. The quite different surface morphology of sample as shown in Fig. 7 is considered to be lead by the various aluminum hydroxides, and influence the outgassing quantities among the samples. We should try near future to understand this problem in detail.

\subsection{Humidity cabinet test}

In the case of EP sample, because of the thick surface oxide layer it showed a good corrosion resistance. CP and other samples in the low outgassing group had less resistance than EP because the thickness of the oxide layer were one order less than the EP sample. But when $25 \mu \mathrm{m}$ of the specimen surface was chemically polished as a special treatment, the change-color-time was increased to $70 \mathrm{~h}$, whereas the thickness of the surface oxide layer was almost the same as that of the standard CP sample by AES.

The humidity cabinet test is one of an accelerated test of corrosion resistant to the atmosphere including moisture. As another problem the change of the outgassing character due to aluminum surface change by atmospheric corrosion was examined. For about three years after the final treatment samples were kept in a room without air conditioning where the temperature and relative humidity changed between 5 and $35^{\circ} \mathrm{C}$, and 30 and $90 \%$, respectively, with seasons and then they were measured with TDS.

$\mathrm{CP}, \mathrm{EP}, \mathrm{MM} 1$, and EX samples were examined. $\mathrm{EL}$ and EX is known to form thin and dense surface oxide layers in common. It had been confirmed by TDS that the outgassing of EX sample is almost the same as that of EL with as received samples. In this after-three-years-measurement, EX was tested as a typical surface treatment to have a thin and dense surface oxide layers. After three years air exposure only the CP samples show the same thermal desorption spectra to that of the as treated ones, whereas thermal desorption spectra of all the other treatments samples were changed. The outgassing quantity $Q$ of before and after air exposure of CP, EX, and MM1 are compared in Fig. 10.

It is presumed that the chemical polishing treatment includes works to change the surface structure of the oxide layer to cause good resistance, though it couldn't be detected by AES. Additional surface analyses are under examination and the results will be reported in near future.

Although not all the origins of the phenomena have been clear yet, practical application of chemical polishing technique to equipments has started.

\section{Summary}

Chemical polishing and subsequent cleaning was proposed as a new technique of aluminum alloys surface treatment applicable at the final stage of production process of chamber and other components of vacuum equipments. Comparing with the material before the treatment the resultant material showed three excellent properties at the same time; 1. the outgassing rate of water is lower than EP, and at the ultimate pressure that is low enough to be used in extremely high vacuum application, 2 . higher corrosion resistance at atmospheric condition than those previously reported treatments with low outgassing rate, and 3. more shiny surface by the reduced roughness. In addition the technique is easy to treat even three dimensional structure without special arrangement, and the uniformity of the properties all over the treated surface, especially for complicated three dimensional parts, are important from the practical point of view.

Although not all the origins of the phenomena have been clear yet and hence further examination is necessary, practical application of chemical polishing technique to equipments has started.

\section{Acknowledgments}

The author would like to thank Professor Y. Tuzi for helpful discussions, Ms. Y. Sato for pump-down experiment and SEM, Dr. Y. Ohtsuka and Ms. Y. Taniuchi for AES analysis and Ms. M. Kusano for BET measurements. 


\section{Reference}

1) H. Ishimaru: J. Vac. Sci. Technol., A2 (1984) 1170.

2) M. Suemitsu, T. Kaneko and N. Miyamoto: J. Vac. Sci. Technol., A5 (1987) 37.

3) A. G. Mathewson, E. Alge, O. Grobnor, R. Souchet and P. Strubin: J. Vac. Sci. Technol., A5 (1987) 2512.

4) N. Kaufherr, A. Krauss, D. M. Gruen and R. Nielsen: J. Vac. Sci. Technol., A8 (1990) 2849.

5) S. Inayoshi, K. Saito, S. Tsukahara, K. Ishizawa, T. Nomura and M. Kanazawa: J. Vac. Soc. Japan, 38
(1995) 199. K. Ishizawa, T. Nomura, A. Shimada, M. Kanazawa, S. Inayoshi, K. Saito and S. Tsukahara: submitted to J. Vac. Soc. Japan.

6) S. Brunauer, P. H. Emmett and E. Teller: J. Amer. Chem. Soc., 60 (1938) 309.

7) J. R. Chen, K. Narushima, M. Miyamoto and H. Ishimaru: J. Vac. Sci. Technol., A3 (1985) 2200.

8) K. Saito, Y. Sato, S. Inayoshi, Y. Yang and S. Tsukahara: J. Vac. Soc. Japan, 38 (1995) 449.

9) K. Watanabe, M. Sasaki, M. Mohri and T. Yamashina: J. Nucl. Mater, 79/77 (1978) 235.

10) W. A. Cannon: Nature, 197 (1963) 1000. 